Wissen, Kommunikation und Gesellschaft.

Schriften zur Wissenssoziologie

Herausgegeben von

Hans-Georg Soeffner

Ronald Hitzler

Hubert Knoblauch

Jo Reichertz

Wissenssoziologinnen und Wissenssoziologen haben sich schon immer mit der Beziehung zwischen Gesellschaften, dem in diesen verwendeten wissen, seiner verteilung und der Kommunikation (über) dieses Wissen(s) befasst. Damit ist auch die kommunikative Konstruktion von wissenschaftlichem wissen Gegenstand wissenssoziologischer Reflexion. Das Projekt der Wissenssoziologie besteht in der Abklärung des Wissens durch exemplarische Re- und Dekonstruktionen gesellschaftlicher Wirklichkeitskonstruktionen. Die daraus resultierende Programmatik fungiert als Rahmen-Idee der Reihe. In dieser sollen die verschiedenen Strömungen wissenssoziologischer Reflexion zu wort kommen: Konzeptionelle überlegungen stehen neben exemplarischen Fallstudien und historische Rekonstruktionen stehen neben zeitdiagnostischen Analysen.

\section{Norbert Schröer \\ Oliver Bidlo (Hrsg.)}

\section{Die Entdeckung des Neuen}

Qualitative Sozialforschung als Hermeneutische

Wissenssoziologie 
Bibliografische Information der Deutschen Nationalbibliothek

Die Deutsche Nationalbibliothek verzeichnet diese Publikation in der

Deuten

<http://dnb.d-nb.de> abrufbar.

\section{Inhaltsverzeichnis}

Oliver Bidlo, Norbert Schröer

Einleitung: Das , abduktive Subjekt' in Wissenschaft und Alltag

\section{TeIL I Die MöglichKeIt des NeueN}

Thomas S. Eberle

Abduktion in phänomenologischer Perspektive

Oliver Bidlo

Kreativität, Abduktion und das Neue. Überlegungen zu Peirce’ Konzeption

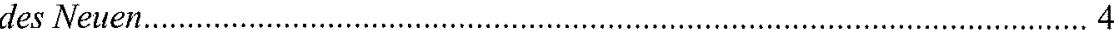

Reiner Keller

Zur Dringlichkeit von Überraschungen.

Ronald Kurt

Improvisation als Methode der empirischen Sozialforschung .....

C) VS verlag für Sozialwissenschaften | Springer Fachmedien wiesbaden GmbH 2011

Lektorat: Katrin Emmerich

vs Verlag für Sozialwissenschaften ist eine Marke von Springer Fachmedien.

springer Fachmedien ist Teil der Fachverlagsgruppe Springer Science+Business Media.

www.vs-verlag.de

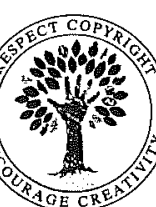

Das werk einschließlich aller seiner Teile ist urheberrechtlich geschützt. Jede Verwertung außerhalb der engen Grenzen des Urheberrechtsgesetzes is ohne Zustimmung des Verlags unzulässig und strafbar. Das gilt insbesondere für Vervielfältigungen, übersetzungen, Mikroverfilmungen und die Einspeicherung und Verarbeitung in elektronischen Systemen.

Die Wiedergabe von Gebrauchsnamen, Handelsnamen, Warenbezeichnungen usw. in diesem Werk berechtigt auch ohne besondere Kennzeichnung nicht zu der Annahme, dass solche Namen im Sinne der Warenzeichen- und Markenschutz-Gesetzgebung als frei zu betrachten wären und daher von jedermann benutzt werden dürften.

Umschlaggestaltung: KünkelLopka Medienentwicklung, Heidelberg

Gedruckt auf säurefreiem und chlorfrei gebleichtem Papier

Printed in Germany

ISBN 978-3-531-17240-8
Norbert Schröer

Not macht erfinderisch. Zur sozialen Praxis , instinktiver Abduktionen ' in

Qualitativer Sozialforschung....

Hubert Knoblauch

Alfred Schütz, die Phantasie und das Neue. Überlegungen zu einer

Theorie des kreativen Handelns.

Andreas Ziemann

Handlung und Kommunikation - eine situationstheoretische

Reformulierung......

Hans-Georg Soeffner

Zwischen Krieg und Frieden - Oder: Der Mensch bemïht sich. Weiß die

„Struktur" es besser?. 


\section{TeIl II DAS NEUE IN DER ALLTÄGLICHEN LEBENSWELT}

Christoph Maeder, Achim Brosziewski

Ethnosonographie: Ein neues Forschungsfeld fïr die Soziologie?

Ronald Hitzler, Michaela Pfadenhauer

Epiphaniebasierte Medizin? Zur Konstruktion diagnostischer Gewissheiten

in der Fernsehserie ,Dr: House"...

Andreas Dörner, Ludgera Vogt

Inszenierung und Kontingenz. Das „Neue“ als Produkt von

kommunikativen Kollisionen

Harald Dern, Christa Dern

Kontingenz in der Fallanalyse. Über den Umgang mit unsicherem Wissen

in der Polizeipraxis.

\section{Joachim Kersten}

Ein Geschehnis im Kiez und ein paar Einsichten im Streifenwagen und in

der Polizeiwache.

Ulrike Froschauer, Manfred Lueger

Das Unmögliche ermöglichen: Zur , rationalen' Konstruktion

,irr-rationaler'Beratungsallmacht.

Sylvia Marlene Wilz

Ansprache und Auswahl. Die Macht der Worte und der Entscheidung in

Prozessen der Organisationsberatung

Autorinnen und Autoren. 


\title{
Abduktion in phänomenologischer Perspektive
}

\author{
Thomas S. Eberle
}

Peirces Abduktion ist berühmt geworden als Verfahren zur Entdeckung des Neuen. Die Phänomenologie hat sich bisher nicht sonderlich für dieses Thema interessiert, ihr ging es vielmehr um eine Konstitutionsanalyse des unbefragten Bodens der Lebenswelt, jenes Sinnfundaments also, das wir stets als selbstverständlich gegeben voraussetzen. Eine phänomenologische Konstitutionsanalyse müsste eigentlich jene Sinnschichten freilegen, in denen sowohl das ,Alte" als auch das „Neue“ konstituiert werden. Neues kann es nur auf der Grundlage von Altem geben. Was soll denn so anders sein, ob man Altes rekonstituiert oder Neues konstituiert? Konstituiert sich „Neues“ etwa auf genuin andere Weise als ,Altes“?

\section{$1 \quad$ Die Entdeckung des Neuen}

1.1 Was ist "neu"?

Das Thema des vorliegenden Bandes, Die Entdeckung des Neuen, wirft unweigerlich die Frage auf, was denn eigentlich als „neu“ gelten darf? Googelt man das Stichwort "neu“, stößt man auf eine Vielzahl neuer Regelungen und Bestimmungen: veränderte Regeln, andersartige Prozeduren, modifizierte Formulare usw. Als „neu“ wird oft das bezeichnet, was ,anders“ ist - anders als das, was vorher war. Eine Änderung bisheriger Routinen wird von den Teilnehmenden eines Settings wohl oft als ,neu“ empfunden - zumindest von jenen, die sich nicht daran erinnern, dass man eventuell das als „neu“ Propagierte schon mal früher institutionalisiert hatte, und daher nicht erkennen, dass man jetzt offenbar zu jener ,alten“ Lösung zurückkehrt. Solches kann man etwa bei manchen Hochschulreformen beobachten, auch etwa bei größeren Unternehmen, die beispielsweise in zyklischen Bewegungen von Zentralisierung auf Dezentralisierung umstellen, nach wenigen Jahren aber wieder auf Zentralisierung, später wieder auf Dezentralisierung usw. Die „Entdeckung des Neuen“ wird dann unter der Hand zur „Wiederentdeckung des Alten“. Was als „neu“ gilt, hat also einerseits mit der lokalen Logik eines konkreten sozialen Settings und seinem Bezugssystem zu tun, und andererseits mit der Zeitspanne des aktualisierten kollektiven Gedächt- 
nisses. Lokal kann auch etwas als „neu“ gelten, das andernorts bereits üblich ist. Ein beträchtlicher Teil dessen, was lokal als „neu“ gilt, wurde dort nicht neu erfunden, sondern ist das Resultat eines sozialen Verbreitungsprozesses, also einer Wissens- und Innovationsdiffusion. Jede Orts- oder Vereinsgeschichte bietet hierzu reichliches Anschauungsmaterial: Oft werden da Personen heroisch für Neuerungen gefeiert, die andernorts schon gang und gäbe waren.

Es mag für die historische Forschung reizvoll sein, jenen Ort und Zeitpunkt aufzuspüren, wo Neues erstmals in Erscheinung getreten ist. Vielleicht empfiehlt es sich auch, in diesem Kontext zu unterscheiden zwischen „Entdeckung“ und „Erfindung“. Seit dem 16. Jh. meint „,ent-decken“ „Unbekanntes, Verborgenes auffinden" (Duden Bd. 7, 1989: 157), was sich in zeitnahen Formulierungen wie der „Entdeckung der Neuen Welt", den „großen Entdeckern" und den „Entdeckerreisen" äußert. Meistens wurde aber etwas „ent-deckt", das - zumindest im naiven Realismus des Alltagsdenkens - schon vorher da war, sei es Amerika, sei es ein bislang unbekannter Planet, seien es die Aborigines in Australien. Die Unterscheidung ,bekannt/unbekannt" verweist unweigerlich auf bestehende Wissensbestände und damit auf soziale Bezugsrahmen: Was den einen unbekannt ist, mag anderen bereits bekannt sein. Dass Kolumbus in Europa als „Entdecker Amerikas" gepriesen wurde, bezeichnete Sorokin bekanntlich als „Christoph Columbus Complex" - es hatten dort ja seit Urzeiten bereits viele Generationen von Menschen gelebt, denen ihr Land hinlänglich bekannt war. Die menschliche Kulturgeschichte ist aber nicht nur eine Geschichte der Entdeckungen, sondern auch eine Geschichte der Erfindungen. Wenn es nicht um die „Entdeckung" von bislang Verborgenem, sondern um die Kreation von Neuem geht, ist „Erfindung“ wohl das treffendere Wort. Interessanterweise erzählt uns die Geschichtsschreibung, dass es Erfindungen gab, die unabhängig voneinander zeitgleich an verschiedenen Orten gemacht wurden (z.B. die Erfindung der Grenznutzenlehre in der Ökonomie von Menger in Wien, von Pareto und Walras in Lausanne sowie von Jevons in Cambridge um 1870 herum). Die Forschung hat in diesem Zusammenhang natürlich immer mit einer unvollständigen Quellenlage zu kämpfen, aber es wird wohl unbestritten sein, dass man - zwar nicht im Einzelfall, aber grundsätzlich und zumindest common-sensically - „Neues“" identifizieren kann, das zu einem bestimmten Zeitpunkt der Kulturgeschichte und an einem bestimmten Ort zum ersten Mal auftrat.

Neues kann also nicht nur entdeckt, sondern auch erfunden werden. Im Alltagsdenken ist die Unterscheidung von Entdeckung und Erfindung fest verankert, wenn sich die semantischen Felder dieser Begriffe an den Rändern auch überlappen. In einer konstruktivistischen Perspektive wird die Unterscheidung allerdings problematisch. So können beispielsweise für Watzlawick (1981) nur die Naturwissenschaften entdecken (nämlich die Wirklichkeit erster Ordnung), während die Menschen ihre sinnhafte soziale und kulturelle Wirklichkeit stets „erfinden“ (Wirklichkeit zweiter Ordnung). Verzichtet man auch auf die Unterscheidung zwischen Wirklichkeit erster und zweiter Ordnung und betrachtet man jede sinnhafte Wirklichkeit als konstruiert, so erweist sich die Unterscheidung von, ,alt" und „neu“ ebenso als Konstruktion. Die für uns entscheidende Frage ist dann, ob wir sie - gemäß der Unterscheidung von Alfred Schütz - als eine Konstruktion erster Ordnung behandeln, also als Typisierung des Alltagsdenkens und damit als unseren Forschungsgegenstand behandeln, oder ob wir sie als Konstruktion zweiter Ordnung, also als Bestandteil des wissenschaftlichen Begriffsapparats verwenden. Diesen Entscheid bewusst und mit Bedacht zu fällen, scheint mir wesentlich, da die Unterscheidung von ,alt" und „neu“ unweigerlich mundane Geltungssetzungen impliziert, die nicht unreflektiert in die wissenschaftliche Perspektive mit aufgenommen werden sollten.

\subsection{Verfahren zur Entdeckung bzw. Erfindung von Neuem}

Wie entsteht Neues? Darüber existiert bereits eine ausgedehnte Forschungs- und Ratgeberliteratur, sei es über die Bedingungen und die Förderungsmöglichkeiten menschlicher Kreativität, sei es zum Innovationsmanagement von Unternehmen. In dieselbe Richtung zielt der neuere Trend in der Organisationsforschung, die "reflective practices" in professionellen Feldern und Organisationen zu erforschen, was in den (verschiedenartigen) Konzepten von „Reflexivität“ auf theoretischer Ebene eine interessante Entsprechung findet. Der große Teil der Publikationen übernimmt die alltagsweltliche Unterscheidung von ,alt“ und „neu“ unreflektiert, ein beträchtlicher Teil besteht in Ratgeberliteratur mit schwacher empirischer Basis, nur ein kleiner Teil erforscht die Kreation von Neuem empirisch und versucht daraus Erkenntnisse für Kreativitäts- und Innovationsförderung zu generieren. In einer groben Systematik können folgende Ebenen unterschieden werden:

a) Auf der organisationalen Ebene geht es darum, Neuerungen der verschiedensten Art einzuführen: Produkt- und Prozessinnovationen, technische und soziale Innovationen, Organisations- und Kulturveränderungen usw. Ein verbreitetes Verfahren ist erstens, von der Konkurrenz zu lernen, um im Wettbewerb bestehen zu können. Neues kann zweitens auch von Unternehmen anderer Branchen gelernt und analog auf die eigene Firma übertragen werden; die Betriebswirtschaftslehre fördert gezielt die Verbreitung solcher Kenntnisse (vgl. etwa den „best methods“ Hype der 1990er Jahre). Drittens kann ein Unternehmen auch gezielt eigene Innovationen entwi- 
ckeln und versuchen, sich am Markt als Innovationsführer zu etablieren. In diesem Fall kommt es nicht umhin, organisationale Bedingungen zu schafdiesem Fall kommt es nicht umhin, organisationale Bedingungen $z$.

b) Die klassischen Kreativitätstechniken leiten an, die Ideenfindung auf individueller Ebene zu entfalten. Spätestens seit den 1950er Jahren, nämlich seit der von Alex F. Osborns entwickelten Methode des „Brainstormings“ gehört es zum Allgemeinwissen, dass kreative Leistungen auch auf der sozialen Ebene gefördert werden können. Das Brainstorming soll ja gerade deshalb so erfolgreich sein, weil ein regelgeleiteter Gruppenprozess in kurzer heit wesentlich mehr Ideen generiert als ein Einzelner dies könnte: Jede Zeit wesentlich mehr Ideen generiert als ein Einzelner dies konnte: Jede einzelne Ideenäußerung kann zahlreiche neue Assoziationen bei den Anderen auslösen und einen wahren Schneeballeffekt auslösen. Inzwischen is die Menge von Kreativitätstechniken fast unüberschaubar geworden, es gibt eher intuitive, assoziationsgeleitete Verfahren neben logisch-systematischen, und solche für Individuen als auch solche für Gruppen, und von beidem zahlreiche Mischformen.

Kreativitätstechniken und „optimale" organisationale Bedingungen können die Kreativitätstechniken und „optimale " Erfindung bzw. Entdeckung von „Neuem“ zwar begünstigen, aber nicht garantieren. Sie bilden also keine hinreichenden, ja vielleicht nicht einmal notwendige Bedingungen. Viele Erfinder erzählen, die entscheidende Idee sei ihnen in irgendeiner Alltagssituation zugefallen, auf dem Klo, im Tram oder beim Tennisgendelen. In der Alltagswelt halten sich denn auch hartnäckig Begriffe wie ,geniaspielen. In "“ ler Einfall", „zündende Idee“, ,göttlicher Funke" als auch die Sozialfigur des Genies", auch wenn diese Termini im wissenschaftlichen Diskurs allesamt bereits seit dem 19. Jahrhundert als obsolet gelten. Dem Alltagsverstand jedoch leuchtet ein, dass es Akteure gibt, die ,mehr" oder ,genialere" oder „kreativere" Einfälle haben als andere, und dieser Topos wird in zahlreichen heroisierenden Erzählungen von großen Erfindern, Künstlern und Wissenschaftlern auch kultiviert und perpetuiert.

http://www.creajour.de/methodisches/technikglossar/index.html

\section{Abduktion nach Peirce}

2.1 Abduktion als logische Operation

Auf diesem Hintergrund überrascht es, dass Charles Sanders Peirce die Entdeckung von „Neuem“ auf ein logisches Verfahren zurückführte: die Abduktion. Ist die Erfindung bzw. Entdeckung von „Neuem“ letztlich eine Frage der Logik? Kann man abduktives Schließen auf dieselbe Art und Weise erlernen wie induktives oder deduktives Schließen? Bräuchten Kreative statt einem Kit von Kreativitäts-Tools vielmehr ein Training in Logik?

Peirce, der sowohl von Russell (1959: 276) als auch von Eco (1989: x-xi) als ,größter amerikanischer Denker" gepriesen wurde, hat sich als Philosoph wie auch als experimenteller Wissenschaftler eingehend mit der Logik wissenschaftlicher Forschung befasst. Sein Werk ist vielschichtig und war in dauernder Entwicklung begriffen, und so überrascht es nicht, dass sich viele PeirceInterpreten untereinander nicht einig sind. Divergierende Auffassungen gibt es auch in Bezug auf die hier interessierende Abduktion, und zwar insbesondere bezüglich der Frage, ob es sich dabei um eine logische Operation handle.

Dieser Ansicht ist zumindest der frühe Peirce, dessen Frühphilosophie mit folgenden Prämissen beschrieben werden kann (Reichertz 2003: 27):

- „Es gibt nur eine Art erkennenden Denkens.

- Erkenntnis ohne vorhergehende Erkenntnis ist unmöglich.

- Der Erkenntnisprozess im Organismus kann als syllogistischer Prozess aufgefasst werden.

- Das Erkenntnisvermögen ist Ergebnis der Evolution der menschlichen Gattung."

Erkenntnis baut mit anderen Worten nicht nur ontogenetisch, sondern auch phylogenetisch auf vorangegangener Erkenntnis auf. Und es gibt nur eine Art erkennenden Denkens, und diese kann syllogistisch beschrieben werden. Peirce, der sich vor allem für Kreativität interessiert, schließt die Möglichkeit von Intuition aus - es gibt keine non-diskursive, vorprädikative Unmittelbarkeit. Erkenntnis ist stets diskursive Erkenntnis und daher ein Prozess gültigen Schließens (Reichertz 2003: 26). So beschreibt Peirce bereits 1868 drei idealtypische logische Schlussverfahren - Deduktion, Induktion und Hypothesis - und führt den Nachweis, dass die Entdeckung von Neuem weder durch Deduktion noch Induktion möglich ist, sondern allein durch Hypothesis (später spricht er von Abduktion). Alle drei Schlussverfahren beschreibt er in Form von Syllogismen, also als Dreischritt 
bei dem von zwei bekannten Größen auf eine unbekannte geschlossen wird: bei der Deduktion als Anwendung allgemeiner Regeln auf besondere Fälle, bei der der Deduktion als Anw von Fall und Resultat auf die Regel und bei der Hypothesis als Schluss von Regel und Resultat auf einen Fall (Peirce 1976: 230ff. - zit.n. Rer Illustration der drei Schlussverfahren durch sein Reichertz 2003: 28). Peirces Illustration der drei Schlussverfahren durch sein duktion als eine logische Operation ausweisen wollen.

Mit dem Begriff Abduktion bezeichnet Peirce später jenen Prozess, durch den Neues entdeckt wird und Hypothesen generiert werden. Die moderne Wissenschaftstheorie verbannt diesen Prozess in den Bereich der Psychologie oder Wissenssoziologie. Damit verpasst sie es gemäß Génova (1997: 117), die Bi1dung von Hypothesen und die Entwicklung wissenschaftlicher Theorien als logisches Problem zu begreifen. Genau dies leiste aber Peirces Untersuchung sches Problem zu begreifen. Genau dies pretation entsprechend von der ,Logik der Überraschung (logic of surprise)“. Weder das Aristotelische Staunen noch der Cartesianische Zweifel genügen als Weder das Aristotelische Staunenten Anstoß geben nach Peirce vielmehr Überraschungen. Ein überraschendes Faktum, das im Rahmen der eingespielten Glaubenssysteme und Ordnungsschemata nich plausibel erklärt werden kann, erfordert einen Bruch mit bisherigen Überzeugungen (habits of belief) und eine neuartige Erklärungsweise, die das Faktum rational verständlich (reasonable) macht. Abduktionen haben nach Nubiola (2005: 126) daher immer eine - und immer dieselbe! - logische Struktur:

„The surprising fact, $\mathrm{C}$, is observed;

But if $A$ were true, $C$ would be a matter of course,

Hence, there is reason to suspect that $A$ is true."

Abduktion als logisches Verfahren zur Generierung von Hypothesen und neuen Theorien ist eine bestechende Idee. Nachdem Popper streng zwischen der Logik der Entdeckung und der Logik der Rechtfertigung unterschieden hatte und nur letztere, in Form deduktiver Theoriesysteme und des Testens von Hypothesen, für wissenschaftstheoretisch legitim hielt, gab es im szientifischen Wissenschaftsverständnis nur noch die Deduktion, und sowohl die Entstehung neuer Ideen als auch induktive Verfahren wurden als nicht- oder vor-wissenschaftlich abqualifiziert. Mit den interpretativen Forschungsansätzen und der davon inspirierten qualitativen Sozialforschung erlebte die Induktion seither eine gewisse Renaissance, und die (lange unbeachtete) Abduktion ersetzt die eingespielte Dichotomie nun durch eine dreistufige Erkenntnislogik: Die Abduktion sucht nach (neuen) Theorien, die Deduktion nach Voraussagen, die Induktion nach Fakten:
„Besteht die erste Stufe des wissenschaftlichen Erkenntnisprozesses in der Findung einer Hypothese mittels Abduktion, dann besteht die zweite aus der Ableitung von Voraussagen aus der Hypothese, also einer Deduktion, und die dritte in der Suche nach Fakten, welche die Vorannahmen ,verifizieren', also einer Induktion. Sollten sich die Fakten nicht finden lassen, beginnt der Prozess von Neuem, und dies wiederholt sich so oft, bis die , passenden' Fakten erreicht sind“" (Reichertz 2003: 96).

Wie jedoch kann man Abduktion als methodisches Verfahren erlernen, so wie man deduzieren und induzieren lernt? Geht es beim Generieren neuer Hypothesen tatsächlich um ein logisches Schließen? Die Antwort auf diese Frage hängt von den philosophischen Prämissen ab, mit denen man operiert. In Bezug auf Peirces Frühphilosophie wurden sie oben schon dargelegt. Wer davon ausgeht, dass Intuition unmöglich ist und Erkenntnis nie vorprädikativ, sondern nur prädikativ und diskursiv sein kann, kommt wohl zwangsläufig zur Auffassung, dass Abduktion - so wie Deduktion und Induktion - ein Schlussverfahren darstellt und als Syllogismus beschreibbar ist. Die entscheidende Frage ist jedoch, ob abduktives Schließen lediglich ex post als logischer Syllogismus rekonstruiert wird, oder ob es bereits als Prozess in Form einer logischen Operation abläuft. Auch für Proponenten der logischen Form von Abduktionen ist Logik allein nicht ausreichend: In der Praxis erweise es sich als schwierig - so Nubiola - Logik mit dem wirklichen Leben zu verbinden. Der Sprachphilosophie des 20. Jh. sei es primär darum gegangen, für die Logik einen Kontext zu liefern. Auch sie reiche jedoch nicht aus - Peirce gehe es darum herauszufinden, wie die wirklichen Prozesse bei Menschen ablaufen, die neue Ideen entwickeln und neues Wissen entdecken (Nubiola 2005: 117). Peirce beschrieb, wie sich abduktive Vermutungen wie Blitze einstellen, als plötzliche Einsichten. Sie geschehen dauernd, im Alltagsleben wie im wissenschaftlichen Forschungsprozess, und weisen dabei eine erstaunliche Effizienz auf: Menschen mutmaßen nicht einfach drauf los, sie würfeln nicht, sondern sie raten erstaunlich erfolgreich. Diese beobachtbare Effizienz ist ihrerseits erklärungsbedürftig. Peirce spricht von einer ,,instinktiven Fähigkeit" (CP 6.476, 1908), und da diese gleichzeitig rational ist, schlägt Ayim (1974: 42) vor, sie einen ,rationalen Instinkt" zu nennen. Nach Nubiola (2005) sieht Peirce, wie sein Vater Benjamin, den Menschen durchwirkt vom göttlichen Geist, von Gott dem Schöpfer, und so beschäftigte er sich auch mit der "Abduktion von Gott" (vgl. Nubiola 2003). Peirce deutet diesen Instinkt aber auch evolutionstheoretisch-pragmatisch und damit säkularer (allerdings letztlich nicht weniger metaphysisch): „man's mind must have been attuned to the truth of things in order to discover what he has discovered. It is the very bedrock of logical truth" (CP 6.476, 1908). 


\subsection{Die abduktive Haltung nach Reichertz}

Es ist das Verdienst von Jo Reichertz (1991, 2003), sorgfältig herausgearbeitet zu haben, dass Abduktion bei Peirce weit komplexer ist als bloß eine logische Operation. Abduktionen lassen sich nicht durch ein operationalisiertes Verfahrensprogramm willentlich herbeizwingen, sondern sie ereignen sich blitzartig, und sie richten sich nicht nach den Gesetzen der formalen Logik (Reichertz 2003: 58). In einer instruktiven Liste stellt er die vielen Bestimmungen der Abduktion im Werk von Peirce zusammen, insoweit sie für die sozialwissenschaftliche Forschung relevant sind (60). Gleichzeitig kritisiert er all jene, die den Abduktionsbegriff einseitig rezipieren und ihn dadurch ausdünnen: Erstens die Auffassung, es handle sich bei der ,Abduktion um eine streng logische Operation" (61); zweitens jene Formulierungen, die besagen, "Abduktion würde Überraschendes erklären und Unverständliches verstehen lassen“ (62) und abduktive Schlussfolgerungen würden ,die beste bzw. die wahrscheinlichste Erklärung liefern“ (63). Stattdessen skizziert er einen „idealtypischen Handlungstypus als Ausdrucksgestalt einer abduktiven Haltung (...): der abduktive Schlussfolgerer ist neugierig, er beobachtet viel und intensiv, stets bereit, sich überraschen zu lassen" (66)

Wie kommt Reichertz zu diesem Ergebnis? Erstens zeigt er auf, dass Peirce im Laufe der Werkentwicklung immer wieder neue Begriffe einfuihrt und alte völlig anders verwendet. Im Unterschied zu seinem frühen Aufsatz, in dem er Induktion und Hypothesis als kenntniserweiternd charakterisiert, schreibt der späte Peirce diese Funktion allein noch der Abduktion zu. Damit grenzt er sie gegenüber der „Hypothesis“ in seinen früheren Arbeiten deutlich ab: ,allein die Abduktion ist kreativ" (Reichertz 2003: 44). Die Abduktion steht nun am Beginn des Erkenntnisprozesses und führt zum Finden einer Hypothese, die anschlieBend getestet wird. Zweitens entdeckt Reichertz, dass Peirce abduktives Schlussfolgern bereits auf vorprädikativer Ebene wirken sieht. Reichertz (2003: 44-52) folgend, möchte ich diesen Aspekt etwas vertiefen.

"Die Erfahrung“, sagt Peirce (1976: 353), ,ist unsere einzige Lehrerin“. Erkenntnis ist nicht ohne Wahrnehmung möglich, und Peirce entwirft daher eine Wahrnehmungstheorie. Erkenntnis ist auch nicht ohne Kommunikation möglich, und so entwickelt er auch eine Zeichentheorie. Durch Zeichensysteme entwickelte sich in der Evolution das Erkenntnisvermögen der menschlichen Gattung, und über Zeichen schließt sich gegenwärtige Erkenntnis auch an vorangegangene Erkenntnis an. Erfahrung ist mit Wahrnehmung und Kommunikation eng verbunden. Reichertz zufolge unterscheidet Peirce den Wahrnehmungsinhal (percept) vom Wahrnehmungsurteil (perceptual judgment) und entwirft beide als abduktive Schlüsse. Ohne abduktives Schlussfolgern besteht der menschliche
Blick aus einem leeren Starren (vacant staring) auf eine ungeordnete Mannigfaltigkeit von Farben und diffusen Formen; erst ein Wahrnehmungsurteil konstruiert eine Ordnung und lässt beispielsweise eine Azalee in voller Blüte sichtbar werden. Wahrnehmungsinhalte sind Bilder, Wahrnehmungsurteile dagegen Texte. Die ,percepts“ bilden die (wahrgenommene) Realität, während Wahrnehmungsurteile Propositionen $\mathrm{zu}$ solchen Wahrnehmungsinhalten sind Zwei Schritte sind nun zu unterscheiden: Wie konstituieren sich die „percepts", und wie kommt man vom Vorprädikativen zum Prädikativen?

Peirce führt dazu drei Ebenen ein: Firstness, Secondness and Thirdness. Auf der ersten Ebene unterscheidet er Empfindung (feeling) und Sinneseindruck (sensation). Beide sind dem Organismus als Ergebnis der Evolution der Gattung einfach gegeben. Während ,feeling“" vollkommen unstrukturiert ist, leistet ,sensation" eine erste Strukturierung, indem sinnliches Unterscheidungswissen (Farbe, Härte, Größe etc.) an die Empfindung herangeführt werden. Auf der zweiten Ebene konstituiert sich das "percept" als Reaktion des Menschen auf ,feelings" und ,sensations". Die ,percepts“ bilden die Realität, den unhintergehbaren Ausgangspunkt aller menschlichen Erkenntnis. Dahinter gibt es keine andere Wel"“ "Percepts" strukturieren wesentlich genauer als Sinneseindrücke, sie enthalten Figuren und Gestalten, und obwohl hier bereits begriffliches Unterscheidungswissen der Gesellschaft zum Tragen kommt, bleiben sie vorsprachlich. Überraschend ist hierbei, dass der Wahrnehmungsinhalt, das ,percept" als „Ergebnis eines Schlusses“ (46) verstanden wird - ja sogar als Ergebnis eines ,abduktiven Schlusses" (45), den ,(d)ie Natur ... ohne Beteiligung menschlichen Bewusstseins" (47) vorgenommen hat. Wenn Abduktion immer „kreativ" zu Neuem führt, liegt offenbar nur dann ein abduktiver Schluss vor, wenn das "percept" fuhrt, liegt offenbar nur dann ein abduktiver Schluss vor, wenn

Wie kommt man nun von der zweiten Ebene zur dritten Ebene: von den vorprädikativen Wahrnehmungsinhalten zu den prädikativen Wahrnehmungsurteilen, vom Unbegrifflichen zum Begrifflichen, vom „Bild“ zum Text? Peirce führt hier den neuen Begriff, ,percipuum" ein, mit dem er den Schritt vom Wahrnehmungsinhalt zum Typus bezeichnet: Die ,percepts“ werden nicht mehr in ihrer konkreten Einzigartigkeit wahrgenommen, sondern als Typus. Wenn ein konkretes „percept“ nicht zu den überlieferten Typen von ,percepts“ passt, löst es Überraschung aus und wird als „neu“ registriert. Dies ist nach Peirce bereits ein Wahrnehmungsurteil, also ein prädikativer Zugriff auf den Wahrnehmungsinhalt und damit der Beginn diskursiver Erkenntnis. Insofern ein „percept“ überrascht, lädt es zu einem kreativen Schluss, also zu einer Abduktion, ein. Peirce versucht mit seiner Wahrnehmungstheorie also zu plausibilisieren, dass das Neue nicht ein körpereigener Prozess ist, sondern von außen kommt und beim Akteur kreative Schlussfolgerungen auslöst. Reichertz betont, 
dass diese "Schlussfolgerungen“ nicht als bewusste Akte menschlicher Vernunft anzusehen sind, sondern dass damit alle bewussten und subbewussten Entscheidungen gemeint sind. Gleichzeitig formuliert er unter Rückgriff auf Wittgenstein, Mead und Piaget diverse Vorbehalte gegenüber der Peirce'schen Wahrnehmungstheorie, die er insgesamt für ,zu idealistisch" (52) hält.

\section{Abduktion in phänomenologischer Perspektive}

\subsection{Die phänomenologischen Ansätze von Peirce und Husserl}

Im Folgenden versuche ich den Beitrag zu eruieren, den die Phänomenologie zur Abduktionsproblematik beitragen kann. Gerade die Ausführungen zur Wahrnen dass es zwischen den Projekten von Peirce und Husserl deutliche Affinitäten gibt. Trotzdem finden sich bisher nur wenige ForHusserl deutliche Affinitaten gie Gemeinsamkeiten und Unterschiede der beiden Ansätze herauszuarbeiten versuchten (Spiegelberg 1956; Mullin 1966; Stjernfeldt sätze herauszuarbeiten ver bedeutendste stammt von Herbert Spiegelberg, der ent2007; Petrilli 2010). Der bedeutendste stammt von Herbert Spiegelberg, der entdeckte, dass auch Peirce den Begriff „Phänomenologie“ verwendete, und dies zum Anlass nahm, die beiden „Phänomenologien“ miteinander zu vergleichen. Peirce benutzte den Begriff allerdings nur für kurze Zeit und ersetzte ihn bald durch andere Begriffe, unter denen „phaneroscopy“ der bekannteste geworden ist. Spiegelberg fand eine interessante Passage in einem Brief von Peirce an Wilist. Spiegelberg fand ein "Phänomenologie“ liam James, wo er diesem seine Position skizzierte: Was er "Phănomenologie nenne, sei eine Wissenschaft, aber radikal verschieden von Psychologie, sie klammere Fragen der Gültigkeit und Ungültigkeit ein und bilde nicht nur die Gogik (Spiegelberg 1956: 168) Grundlage der Philosophie, sondern auch der turaserls Phänomenologie zu, Diss dass Spiegelberg herauszufinden versuchte, ob der Eine vom Ander Kent hatte. In beiderlei Richtungen kam er zu einem negativen Ergebnis: Obwohl beide sich ausgiebig mit Logik befassten und deren Fundierung schließlich in der Phänomenologie suchten, war keiner der beiden je mit dem Anderen in Berührung gekommen.

Neben den überraschenden Übereinstimmungen zwischen den beiden Anvibt es allerdings auch deutliche Differenzen. So verfolgten beide ein unsäzen gibt es allerding die schließlich die „Krisis der Europäischen Wissenschaften“ (Husserl 1954) beheben wollte, indem deren (vergessene) Fundierung in der Lebenswelt mittel transzendentalphänomenologischer Konstitutionsanalysen aufgewiesen wurde. Die Krise bestehe darin, dass das Ideenkleid wissenschaftlicher Theorien für wahres Sein genommen werde, obwohl es lediglich eine Methode sei (1954: 52). Die Phänomenologie soll nun den Sinn menschlichen Daseins durch eine Analyse von dessen Geltungsmodi erschließen und die Wissenschaften systematisch in der Lebenswelt verankern (1954: 171). Peirce hingegen war - so Spiegelberg (1956: 169) - primär an einem Kategoriensystem interessiert, welches das gesamte Wissensuniversum zu ordnen erlaubte. Was Peirce "Phänomenologie“ nannte, hat er nie wirklich systematisch entfaltet und weiterentwickelt. Seine Wahrnehmungstheorie wirkt im Vergleich zu Husserls phänomenologischen Untersuchungen denn auch wenig elaboriert. Das möchte ich im Folgenden näher erläutern.

Ein grundlegender Unterschied zwischen Peirce und Husserl besteht im erkenntnistheoretischen Ausgangspunkt. Husserl (1950, 1971, 1976, 1991) stützt sich auf Brentanos Konzept der Intentionalität und setzt seine Analysen beim ego-cogito-cogitatum an. Phänomene sind daher immer Bewusstseinsphänomene, d.h. sie konstituieren sich durch synthetische Akte eines subjektiven Bewusstseins. Dieses ist aber immer intentional auf etwas bezogen, also ein Bewusstsein-von-etwas. Im Gegensatz zu Descartes gibt es kein blosses ego cogito, sondern das Erkennen, das cogitare, ist immer auf ein cogitatum, ein Erkanntes bezogen. Wahrnehmungsinhalte lassen sich daher - im Unterschied zu Peirce nie von den Wahrnehmungsakten trennen. Die Wahrnehmungsinhalte können sich zwar durchaus auf Gegenstände in der so genannten „Außenwelt" beziehen - doch ist dies für Husserl irrelevant: Mit der epoché klammert er die Geltungssetzungen explizit ein - ob es sich also um „real existierende“ oder „nur eingebildete“ Dinge handelt, ist unerheblich. Husserl interessiert sich allein für die Konstitutionsanalyse von Phänomenen - und zwar von Phänomenen jeglicher Art. Denn real oder nicht - Phänomene haben vieles gemeinsam. Jedes Phänomen bildet beispielsweise immer eine noetisch-noematische Einheit. Wenn sich ein Phänomen verändert, kann dies zum einen durch die noesis, durch die noetischen Bewusstseinsakte bedingt sein: Ein Phänomen gewinnt zum Beispiel schärfere Konturen, indem ich mich ihm mit größerer Aufmerksamkeit zuwende als zuvor. Die Veränderung eines Phänomens kann zum anderen aber auch darin begründet sein, dass sich das noema verändert, das heißt dass sich -vereinfacht ausgedrückt - der wahrgenommene "Gegenstand" selbst wandelt. Wenn ein wahrgenommenes Phänomen also beispielsweise verschwommen und undeutlich ist, kann dies aufgrund der noetisch-noematischen Einheit sowohl darin liegen, dass ich nur flüchtig und oberflächlich hingucke, als auch darin, dass das Wahrgenommene gar nicht deutlicher gesehen werden kann (weil es zum Beispiel im Nebel steckt). Oft interessiert einen das nicht weiter - das Phänomen erscheint mir einfach so, wie es mir erscheint. Durch Variationen meiner Zuwendung kann 
ich aber auch (mehr oder weniger systematisch) erproben, welche Modifikationen noetischer Natur sind und welche im noema selbst begründet sind.

Indem Husserl die unmittelbare Anschauung - das ego-cogito-cogitatum zum Ausgangspunkt und die Gegebenheitsweisen der Phänomene zum Gegenzum Ausgangspunkt und seiner Analysen macht, kann er die Aporien der Peirce'schen Wahrnehstand seiner Analysen macht, kann er die Aporien der Peirce'schen Priorität, wie firstness, secondness and thirdness, sind wenig erhellend - warum auch soll das firstness, secondness and thirdness Eine vor dem Anderen kommen? Stattessen untersucht Hussert die Prozs sinnlich Sinnkonstitution im subjektiven Bewusstsein und erkennt, dass selbst sinnlich wahrgenommene Phänomene sich nicht nur sinnlich (nach Farben, Gerüchen, Tönen usw.), sondern auch sinnhaft konstituieren. Husserl unterscheidet Erfahrung und Urteil, also den vorprädikativen Bereich der inneren Anschauung und das prädikative Urteil $(x=p)$. Phänomene konstituieren sich als sinnhafte schon das prädikative Urteil $(x=p)$. Phannomene konstitueren sich als sinnhafte schon auf der vorprädikativen Ebene, nicht erst dank eines Wahrnehmungsurteils, wie bei Peirce. Welches Phänomen sich im subjektiven Bewusstsein jeweils von anderen abhebt, hängt vom attentionalen Blickstrahl des Betrachters ab, mit andeWorten von seinem jeweiligen Interesse. Jedes Phänomen ist dabei von einem äußeren Horizont umgeben - die Gestaltpsychologie operierte analog mit dem Schema Figur/Hintergrund, und heute würde man statt von „Horizont" eher dem Schema Figur/Hintergrund, und heute würde man statt von "Hortizont wer wird. von „Kontext" sprechen -, der zu den Rändern hin immer undeutlicher wird. Den Gesamthorizont bildet der nicht-hinterfragte Boden der Lebenswelt, also die als selbstverständlich vorausgesetzten Wissensbestände und Glaubensüberzeugungen.

Husserl erkannte, dass Phänomene auch einen inneren Horizont haben, d.h. man sieht" immer mehr als der Wahrnehmung gegeben ist, das Wahrgenommene weist über sich hinaus, oder anders ausgedrückt: Im Wahrgenommenen wird Anderes, Nicht-Wahrnehmbares ,appräsentiert". Betrachtet man ein Haus von Anderes, Nicht-Wahrnehmbares "appras fieht man nur eine Fassade, doch appräsentiert die Fassade gleichzeitig die Rückseite des Hauses, auch wenn letztere im Augenblick nicht gleichzeitig die Rücken sieht von an ein „Haus“ und nicht wahrgenommen werden kann - mail sien phänomenologischen Untersuchungen nur eine „Fassade“. In seinen detaillierten phänomenologischen Untionen“, die er versucht Husserl detaillio Apperzeptionen" nennt, nicht Ergebnis eines Schlusses sind, auch ,analogische Apperzeptonen beruhen. So kann wie bei Peirce, sondern auf passiven Bewusstseinssynthesen beruhen. So kann
eine aktuelle Wahrnehmung in passiver Synthesis auch eine Erinnerung oder eine Phantasievorstellung „wecken“ oder „hervorrufen“, wodurch zwei getrennte Phänomene sich als Einheit konstituieren, ohne dass das Bewusstsein in Form von Schließungsprozessen aktiv beteiligt wäre. Appräsentative Paarungen speisen sich selbstverständlich aus der Erfahrung - dass unsere Städte aus Häusern und nicht aus bloßen Fassaden bestehen, hat sich immer wieder bestätigt
Die Unterscheidung von Erfahrung und Urteil bzw. von vorprädikativer und prädikativer Ebene ist in Husserls Phänomenologie zentral (Husserl 1985). Wahrnehmung vollzieht sich in phänomenologischer Perspektive - im Unterschied zu Peirce - nicht nur auf der diskursiven, sondern auch auf der nondiskursiven Ebene, und entsprechend sucht Husserl - anders als Peirce - Universalien nicht nur auf der prädikativen, sondern auch auf der vorprädikativen Ebene. Prozesse der Typisierung sind nicht an Sprache gebunden, sondern finden als passive Vorkonstruktion des Realen bereits präreflexiv statt - vor jeglicher Symbolisierung oder begrifflicher Explikation. Damit setzt sich die Phänomenologie markant von sprachphilosophischen Ansätzen ab, die letztlich alles auf Sprache zurückführen und semiotisch erklären wollen. Srubar (im Druck) hat minutiös aufgezeigt, dass einerseits Sinnbildung im Prozess hybrider Kommunikation mit Objekten durchaus asemiotischen Charakter haben kann und dass es andererseits auch auf intersubjektiver Ebene asemiotische Kommunikationen sehr bedeutender Art gibt, wie er an den Beispielen von Gewalt und Sexualität überzeugend illustriert. Im Unterschied zu den semiotischen Ansätzen erlaubt die Phänomenologie, auch sinnbildende Prozesse präsemiotischer bzw. asemiotischer Art zu analysieren.

Reichertz ist in seiner Werkexegese zum Schluss gekommen, dass die „abduktiven Blitze“, wie sie der späte Peirce beschrieb, auf vorprädikativer Ebene stattfinden. In der phänomenologischen Perspektive erscheint dies als sehr plausibel: Abduktive Blitze ereignen sich wie Appräsentationen als passive Bewusstseinssynthesen - sie stellen sich ein, indem Phänomene plötzlich in einem neuen, ungewohnten Zusammenhang gesehen und dadurch neu gedeutet werden Dies ist nicht Ergebnis eines Schlusses, wie Peirce meint, sondern Ergebnis einer passiven Synthesis. Husserls Vorschlag, Prozesse (logischen) Schließens auf der prädikativen Ebene zu verorten, auf der vorprädikativen Ebene aber von passiven Bewusstseinssynthesen zu sprechen, eignet sich offensichtlich, die von Reichertz (2003: 23) geschilderte „Kehre“ in Peirces Konzept der Abduktion erkenntnistheoretisch zu verdeutlichen. Peirces Kehre besteht, derart gerahmt, nicht nur darin, dass sich abduktive Blitze nicht auf prädikativer, sondern auf vorprädikativer Ebene ereignen, sondern auch darin, dass sie dadurch auch nicht mehr Ergebnisse logischer Schlussfolgerungen sind, ja radikaler: dass dabei überhaupt keine Prozesse des Schließens mehr involviert sind.

Nach den Beiträgen der Sprachphilosophie im 20. Jh. wird heutzutage wohl niemand die Rolle der Sprache für die Sinnkonstitution unterschätzen wollen. Das Meiste des von uns Wahrgenommenen haben wir auch bereits mal prädikativ thematisiert, ist also auch geprägt durch gesellschaftliche Diskurse und die in diesen vorherrschenden sprachlichen Typen. Husserl hat, im Unterschied zu Peirce, die Relevanz der Sprache wenig beachtet und kaum thematisiert, obwohl 
phänomenologische Untersuchungen einen erwachsenen, also in einer bestimmten Gesellschaft und Kultur sozialisierten Menschen voraussetzen. Können prädikative und vorprädikative Ebene tatsächlich auseinandergehalten werden? Zur Beantwortung dieser Frage muss zunächst geklärt werden, was denn eigentlich unter „Prädikation" verstanden wird. Ein Blick auf die diesbezüglichen Debatten zeigt, dass die Deutung von „Prädikation“" durchaus vielfältig ist. In Bezug auf das Werk Heideggers, der vorgängiges Verstehen ebenfalls als vorprädikativ versteht, identifiziert Henning (2001) drei verschiedene Deutungsarten: Eine erste Gruppe von Interpreten versteht darunter „Sprachlichkeit schlechthin“; eine zweite Gruppe versteht darunter ,,die Bezeichnungsfunktion der Sprache, die von ihrer Urteilsfunktion zu unterscheiden sei“; eine dritte Gruppe meint damit einen „,spezifischen wissenschaftslogischen Sprechakt", nämlich die ,existentiale Fundierung der theoretischen Sprache in einer vortheoretischen". Die drei Auffassungen von „Prädikation" unterscheiden sich beträchtlich und implizieren ebenso deutliche Unterschiede in Bezug darauf, was jeweils unter „vorprädikativ“ zu verstehen ist.

Peirce sprach explizit von einem ,Wahrnehmungsurteil". Aufgrund von Reichertz' Darlegung ist zu vermuten, dass er dabei Bezeichnungs- und Urteilsfunktion der Sprache nicht unterschied, sondern allein schon die Bezeichnung ein „Wahrnehmungsurteil" nannte. Husserl - wie Peirce von der Logik herkommend - verstand unter Prädikation Urteile der Art $x=p$, daher unterschied er „Urteil“" und „Erfahrung“. Gewiss lässt sich plausibel argumentieren, dass sprachliche Typisierungen auch die Form der Erfahrung prägen. Husserls phänomenologische Analyse erschöpft sich aber nicht in Sprachanalyse, sondern bezieht auch Phänomene in die Untersuchung mit ein, die sprachlich schwer fassbar oder sogar vorsprachlicher Natur sind. Die Phänomenologie ist zwar gezwungen, Aussagen über den vorprädikativen Bereich ebenfalls im Medium der Sprache zu formulieren, lässt sich indes nicht zum Fehlschluss verführen, der vorprädikative Bereich sei ausschließlich sprachlich strukturiert. Wie bereits erwähnt, liefert Srubars (im Druck) Analyse der asemiotischen Kommunikation dazu reichhaltiges Anschauungsmaterial und treffliche Argumente.

\subsection{Die Mundanphänomenologie von Alfred Schütz}

Schütz’ Mundanphänomenologie führt Husserls Analysen auf eine Art und Weise weiter, die geeignet ist, den vorprädikativen Prozess der Abduktion noch tiefschürfender zu klären. Schütz übernimmt sämtliche der oben angeführten Untersuchungsergebnisse von Husserl. Darauf gestützt führt er auch feinsinnige Analysen der Sinnkonstitution im subjektiven Bewusstseins, der Einordnung von
Erlebnissen in Erfahrungszusammenhänge, der polythetischen Zeitstruktur und des reflektiven monothetischen Zugriffs, des Fremdverstehens über Zeichen und Anzeichen, des Unterschieds zwischen alltäglicher und wissenschaftlicher Betrachtungsweise und vielem mehr durch (Schütz 2004a) - alles Analysen, die differenzierte und überzeugende Antworten auch auf die Fragen von Peirce bezüglich Erkenntnis, Erfahrung und Wahrnehmung geben. Mit seiner Theorie der Appräsentation, der Transzendenz und der Sprache sowie mit dem pragmatischen Begriff der Relevanz leistet Schütz aber auch eine substantielle Weiterentwicklung der Phänomenologie Husserls und baut gleichzeitig eine Brücke zum Pragmatismus von Peirce: Zum einen übernimmt er anthropologische Grundannahmen sowie das Konzept der relativ-natürlichen Einstellung von Max Scheler, der im Unterschied zu Husserl Peirce rezipierte (Scheler 1926: 259 323). In seinen Vorlesungen zur Sprachphilosophie setzt sich Schütz (2003b: 263ff.) zum anderen auch explizit mit Peirces Theorie des Zeichens auseinander. Wie Srubar $(1988,2007)$ in einer sorgfältigen Werkexegese herausarbeitete lässt sich bei Schütz eine ,pragmatische Wende“ beobachten, die schon vor seiner Emigration in die USA einsetzte. So weist Schïtz' Konzeption von Lebenswelt auch einen intersubjektiven, nicht nur einen subjektiven Pol auf (wie bei Husserl): „Den einen Pol stellt die in Wirkensbeziehungen hervorgebrachte, intersubjektive durch das System der pragmatischen Relevanzen geordnete und dadurch auferlegte soziale Wirklichkeit dar, der andere Pol ist die subjektive Spontaneität, als Quelle der Aneignung und der subjektiven, ,privativen' Modifikation der so auferlegten Realität" (Srubar 2007: 199). Schütz bezieht die Pole aufeinander und fasst sie zu einem Konstitutionsprozess zusammen, die durch appräsentative Systeme vermittelt sind. Damit überschreitet er sowohl die Restriktionen der Transzendentalphänomenologie Husserls als auch jene des Pragmatismus von Peirce.

Schütz baut zugleich eine Brücke zwischen den beiden Ansätzen. Er akzeptiert viele von Husserls transzendentalphänomenologischen Erkenntnissen, doch betreibt er seine eigenen phänomenologischen Analysen „,mundan“, also nicht in der Sphäre transzendentaler Reduktion, wo mittels der epoché die alltäglichen Annahmen und Glaubensüberzeugungen der natürlichen Einstellung eingeklammert werden. Doch auch seine Mundanphänomenologie stößt immer wieder an Grenzen, wo er von der phänomenologischen in eine pragmatistische Perspektive, also vom subjektiven zum intersubjektiven Pol wechselt: Da Menschen von Müttern geboren und von anderen Menschen beeinflusst und erzogen werden, sucht Schütz die Begründung der Intersubjektivität nicht - wie Husserl - in der Phänomenologie, sondern in einer ,interaktionistischen" Betrachtungsweise, nämlich in der sozialen Wirkensbeziehung. Bei der Geburt wird ein Baby in eine bestimmte Gesellschaft und Kultur hineingeboren, von der es sozialisiert wird. 
Das von ihm sukzessive erworbene Wissen ist sozial abgeleitet, und der in einem bestimmten Hier und Jetzt zuhandene Wissensvorrat ist immer biographiebestimmten Tier und Jetzt zuhandene Wissensvont ist imiori, also den obspezifisch komponiert, vom jeweiligen gesellschaftlichen Apriori, also den objektivierten sprachlichen und vorsprachlichen Typisierungen und den gesell-

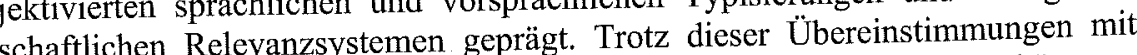
schaftlichen Relevanzsystemen geprägt. Trotz dieser Ubereinstim di Phänomepragmatistischen Positionen (insbesondere zu Mead) gibt Schütz die Phänomenologie nicht auf, sondern betont nachdrücklich, wie wichtig die phänomenolonologie nicht auf, sonterionalen Leistungen des Subjekts für eine Konstitutionsgische Analyse der intentionale

Schütz beginnt die meisten seiner Analysen von einem phänomenologischen Schülz beginn din denen er Ausgangspunkt. Auch seine sprachphilosophischen Eröterungen, in denen er seine Theorie der appräsentativen Systeme entwirft, starten mit Husserls Konzept der Appräsentation, wobei Schütz Appräsentationen nicht als bloße Bezept der Appräsentation, wobei Schütz Appräsentation in einen pragmatischen $\mathrm{Zu}-$ sammenhang stellt. Schütz (2003b: 128ff.) übernimmt alles, was Husserl über Appräsentationen gesagt hat: dass sie keine Analogieschlüsse, sondern passive Appräsentationen gesagt hat: dass sie keine Analogieschlüsse, sondern passive Synthesen darstellen; dass das appräsentierende Element das appräsentierte "weckt" oder "hervorruft"; dass die appräsentierende Erfahrung nicht nur in der Wahrnehmung eines Dinges, sondern auch einer Erinnerung, Phantasievorstellung oder ein Traum sein kann. Mit Hilfe von Bergsons Konzept der „Ordnunlung " Appräsentation zu unterscheiden: Je nachdem, auf welche Wirklichkeitsordnung bzw, auf welchen Wirklichkeitsbereich sich eine Appräsentation bezieht, sprich er von Apperzeptionsschema, Appräsentationsschema, Verweisungsschema und Deutungs Srubar):

Mit dem Apperzeptionsschema wird das wahrgenommen, was der unmittelbaren Apperzeption gegeben ist (ich sehe beispielsweise zwei Balken auf einem Dach, die im rechten Winkel gekreuzt sind).

Mit dem Appräsentationsschema wird der unmittelbar apperzipierte Gegenstand als Glied eines appräsentativen Paares angesehen, die beide demselben Wirklichkeitsbereich angehören (ich sehe die beiden gekreuzten Balken als „Kreuz", also als ein Zeichen).

Schütz unterscheidet zudem zwischen der erkenntnistheoretischen Orientierung des amerikanischen Pragmatismus (James, Peirce, Dewey) auf der einen und der europäischen Tradition pragmatistischen Philosophierens (Bergson und Scheler) auf der anderen Seite. Scheler behauptet eine Priorität des pragmatischen Motives nur auf der mundanen, nicht jedoch auf der erkentnistheoretischen Ebene (Endreß/Srubar 2003). c) Mit dem Verweisungsschema wird etwas appräsentiert, das zu einem anderen Wirklichkeitsbereich gehört (z.B. verweist das Kreuz auf die christliche Religion, auf die Geschichte Jesu und seiner Kreuzigung usw.).

d) Mit dem Rahmen- oder Deutungsschema wird das Verhältnis sichtbar, das zwischen dem Appräsentations- und Verweisungsschema besteht. So ergibt sich die „eigentliche" Bedeutung des Zeichens erst aus dem Kontext eines breiteren Deutungsschemas, in den es eingegliedert wird (z.B. bedeutet das Kreuz-Zeichen für Atheisten etwas ganz anderes als für Protestanten und Katholiken)

Ab c) spricht Schütz (2003b: 134) von Appräsentationsverweisungen „höheren Grades"; sie setzen ,die Kenntnis des Bereichs voraus, innerhalb dessen die Paarung selbst stattfindet". Was nun die vier Appräsentationsformen anbelangt, kann in einer konkreten Appräsentationsbeziehung irgendeines dieser Schemata als Ausgangspunkt bzw. als unser Bezugssystem gewählt werden und sich gegenüber den anderen quasi verselbständigen - die anderen Schemata erscheinen dann jeweils als mehr oder weniger irrelevant.

Auf diesen vier Appräsentationsformen aufbauend entwickelt Schütz eine eigene Sprachtheorie. Dabei untersucht er viele der vorliegenden sprachtheoretischen Ansätze und konstatiert, dass deren Unterschiedlichkeit primär darauf zurückzuführen sei, dass sie an unterschiedlichen Punkten ansetzten - faktisch seien sie aber gar nicht so unterschiedlich, wenn man sie auf einer anderen Grundlage - eben der Appräsentation - betrachte. Schütz verwendet einen mehrstufigen Zeichenbegriff, der Merkzeichen, Anzeichen, Zeichen und Symbole unterscheidet. Dank dieser appräsentativen Systeme werden transzendente Bereiche appräsentiert: Dank der Zeichen (insbesondere Sprache) wird beispielsweise das Bewusstsein des Anderen zugänglich und damit Intersubjektivität ermöglicht, und dank der Symbole werden in der Alltagswelt Wirklichkeiten vergegenwärtigt, die nicht sinnlich erfahren werden können (z.B. religiöse, ,jenseitige" Welten). Die Sprache ist das Appräsentationssystem par excellence, enthält es doch als historisches Zeichensystem eine große Vielfalt von Typisierungen, Abstraktionen und Standardisierungen und damit auch einen Großteil des gesellschaftlichen Wissensvorrates. Für Schütz ist Sprache allerdings mehr als de Saussures langue - im Zentrum steht immer das Sprechhandeln. Wie bei Husserl, geht dieses indes auch bei Schütz über Sprache hinaus: Dazu zählen denn beispielsweise "Ausdrucks- und Nachahmungsbewegungen, Begrüßungsgesten, Gesten der Ehrerbietung, des Respekts usw." (Knoblauch et al. 2003: 27) - kurz: der bereits erwähnte Bereich der asemiotischen Kommunikation. Auch für diesen nichtsprachlichen Bereich gibt es nämlich objektivierte Handlungs- und Deutungsschemata. Auch Schütz' Typisierungslehre reicht daher, auf Husserl aufbauend, 
in den vorprädikativen Bereich hinein (womit auch er die Restriktionen der Sprachphilosophie überschreitet).

In Bezug auf die Unterscheidung von prädikativ und vorprädikativ stützt sich Schütz präzise auf Husserl. Im Unterschied zu diesem betont er indes die zentrale Bedeutung der Sprache. Dabei unterscheidet er Phänomene, die unserer Wahrnehmung in der gesamten sinnlichen Fülle vis-à-vis gegeben sind, und deren sprachlicher Typisierungen, die in ganz unterschiedlichen AbstraktionsAnonymitäts- und Generalisierungsstufen vorgenommen werden können. Sprachliche Aussagen sind entsprechend hoch selektiv. So kann ich eine konkret wahrnehmbare Person als Menschen, als Säugetier, als Mann, als Türke, als Kurde, als Asylanten, als Juristen, als Anwalt, als Politiker, als Guerillakämpfer und vieles mehr bezeichnen; im konkreten Fall kann dies alles auch empirisch richtig sein, doch mit der sprachlichen Typisierung werden aus der vorhandenen Fülle lediglich einzelne Merkmale herausgehoben, wodurch - je nach Relevanzsystem - eine Selektion aus den vielen empirisch zutreffenden Möglichkeiten der sprachlichen Typisierung getroffen wird. Es spricht nun einiges dafür, sprachliche Aussagen über die wahrgenommene Person als „prädikativ“ zu bezeichnen, während die konkrete inhaltliche Fülle der face-to-face wahrgenommenen Person „,vorprädikativ“ ist. De facto wird auch diese Ebene der Wahrnehmung durch Sprache imprägniert sein, trägt doch die Sprache das Sinnkleid der sozio-kulturellen Welt. Die Wahrnehmung vollzieht sich jedoch nicht in sprachlicher Form, besteht nicht in einem Wahrnehmungsurteil wie bei Peirce, sondern bildet einen Komplex mit mannigfaltigen Sinnschichten unterschiedlicher Deutlichkeitsstufen. Der unbefragte Boden der Lebenswelt ist zweifellos auch von Sprache mitgeprägt, schwingt jedoch in seiner Unbefragtheit und Selbstverständlichkeit nur undeutlich - und sicher nicht prädikativ - mit.

Schütz' Analyse der Typisierung steht in einem unmittelbaren Zusammenhang mit seiner Theorie der Relevanz (Schütz 2004b). In dieser unterscheidet er thematische Relevanzen, Auslegungsrelevanzen und Motivationsrelevanzen. Die thematische Relevanz drückt sich in der Frage aus, was thematisiert wird, d.h. worauf die subjektive Aufmerksamkeit gerichtet wird. Entsprechend hebt sich etwas heraus, umgeben von einem nicht-thematisierten Horizont. Im Alltagsleben sind dies nach Schütz typischerweise nicht Routine-Situationen, sondern Situationen, die aus irgendeinem Grund ,problematisch" geworden sind. Mit der thematischen Relevanz eng verbunden ist die Auslegungsrelevanz: nach ihr bestimmt sich, wie ich ein Phänomen - z.B. eine problematische Situation - interpretiere. Die Motivationsrelevanz bezieht sich schließlich auf meine Um-zuMotive, rückblickend auch auf meine Weil-Motive, und manifestiert, warum ich eine Handlung plane, ausführe oder unterlasse. Auch in der egologischen Analyse des Phänomenologen wird deutlich, dass diese Relevanzen nur zum Teil selbst gewählt sind, zum Teil aber auch von außen auferlegt werden. Bereits das gesellschaftliche Apriori der Lebenswelt, in die wir hineingeboren wurden, beinhaltet mannigfaltige fest institutionalisierte Relevanzsysteme, von denen im Laufe des Sozialisationsprozesses eine ganze Reihe internalisiert werden. So kann der biographiespezifische subjektive Wissensvorrat anhand seiner strukturellen Grundkomponenten Typik und Relevanz betrachtet werden: Subjektive Erfahrung, inklusive medial Angeeignetes, ist in typischen Formen sedimentiert, und die zuhandenen Wissensbestände bringen die vergangenen Relevanzen der eigenen Lebensgeschichte zum Ausdruck, seien sie selbst gewählt oder auferlegt worden. Von den biographiespezifischen Relevanzsystemen $\mathrm{zu}$ unterscheiden sind die aktuellen Relevanzen in einem jeweiligen Hier und Jetzt, also das jeweilige pragmatische Interesse in einer konkreten (sozialen) Handlungssituation. Dieses bestimmt, was, wie und warum etwas thematisiert und wie es konkret ausgelegt, d.h. typisiert wird. Diese Relevanzen sind auch ausschlaggebend, wenn es um die Entdeckung des Neuen geht.

\subsection{Abduktion in phänomenologischer Perspektive}

Was für Folgerungen lassen sich nun bezüglich Abduktion aus phänomenologischer Perspektive ziehen? Ein zentraler Unterschied zu Peirce ist offensichtlich, dass überraschende Fakten nicht nur von außen induziert, also noematisch bedingt sein müssen, sondern dass sie auch durch noetische Variationen herbeigeführt werden können: Man entdeckt Neues, indem man Gegebenes anders interpretiert. Ich behaupte sogar, dass die noetischen Akte wesentlich entscheidender sind als das noema. Es gibt Leute, deren Wahrnehmungsmodi derart habitualisiert sind, dass sie Veränderungen in ihrer Umwelt überhaupt nicht mehr wahrnehmen: die neue Frisur, das neue Kleid, der andersfarbige Lippenstift der Gattin bleiben vom Ehemann einfach unbeachtet - man(n) sieht, was man immer sieht. Achtsamkeit ist daher das erste Gebot, wenn Neues entdeckt werden soll: Achtsamkeit für (auch subtile) Unterschiede, für Veränderungen des Gewohnten, für bisher Unbeachtetes. Auch in habitualisierten Wahrnehmungsfeldern lässt sich immer wieder Neues entdecken -Achtsamkeit wirkt horizonterweiternd.

Auch noematisch Konstantes kann neu gesehen werden, indem man es anders interpretiert. Erinnert sei an Husserls Technik der freien Variation, die er zum Zweck der eidetischen Reduktion entwickelte: Man variiert ein Phänomen in verschiedenster Hinsicht, um die Grenzen auszuloten, an denen es in etwas Andersartiges umkippt. Husserl wollte auf diese Weise das eidos, das ,Wesen“ der Phänomene bestimmen. Wenn ich beispielsweise einen Würfel betrachte, kann ich mich fragen, ob es immer noch ein Würfel ist, wenn ich ihn mir größer oder 
kleiner vorstelle, wenn er aus hartem oder weichem Material ist, wenn er anders riecht oder andere Farben hat usw. Ja, bei all diesen Variationen bleibt der Würfel ein Würfel, diese Merkmale sind also keine eidetischen, sie gehören nicht zum Wesen des Würfels. Der Würfel ist jedoch nicht mehr ein Würfel, wenn ich ihn mir mit ungleichen Seitenlängen vorstelle, wenn er nicht sechs Seiten hat, wenn er rund ist usw. Die Technik der freien Variation ist also ein bewusstes, einigermaßen systematisches Vorgehen. Sie lässt sich ebenso einsetzen, wenn man Neues entdecken will: Man variiert ein Phänomen in verschiedenster Hinsicht und versucht, es auf ganz unterschiedliche Weise wahrzunehmen bzw. zu interpretieren.

Auch Schütz' Theorie der Relevanz vermag Anhaltspunkte zu geben, wie man den Wahrnehmungsakt gezielt variieren kann. Eine Variation der thematischen Relevanz mag neue Phänomene sichtbar werden lassen, die vorher unbeachtet oder sogar tabuisiert blieben, eine Variation der Auslegungsrelevanz kann unterschiedliche Interpretationsmöglichkeiten zutage fördern. Dahinter steht natürlich die Motivationsrelevanz, das eigene Interesse, also beispielsweise die Frage, ob man in einer gegebenen (sozialen) Situation überhaupt Neues entdecken will. Seit Goffmans (1977) Rahmenanalyse haben viele durch eine Variation der Rahmen versucht, die Dinge in neuem Licht zu sehen. Goffmans Konzept des „Rahmens" entspricht dabei weitgehend dem Schütz'schen Begriff des „Deutungs- oder Interpretationsschemas" (vgl. Eberle 1991), wobei Goffman eine Mannigfaltigkeit gesellschaftlicher Rahmen-Modifikationen und RahmenTransformationen aufzeigt. Auf Schütz' Anregung könnte man den Versuch wagen, Variationen gezielt anhand der vier Appräsentationsformen durchzufuihren, also auch auf der Ebene der Apperzeption, der Appräsentation und der Verweisung. Allerdings blättern erst Rahmen- und Deutungsschemata die vielschichte Komplexität von Wissensbeständen auf, die in einer Interpretation involviert sind. Abduktionen dürften dann nicht nur durch Variationen und Einklammerungen bisheriger Deutungsgewohnheiten befördert werden; sie werden auch umso mehr ermöglicht, je breiter, tiefer und feingliedriger der (subjektive) Wissensvorrat ist, den sie als Ressource verwenden können.

Abduktive Blitze ereignen sich im Licht phänomenologischer Analysen wohl analog zu Appräsentationen, nämlich als passive Bewusstseinssynthesen, beruhen also nicht auf (logischen) Schlüssen. Appräsentationen beruhen jedoch auf Erfahrung - das Appräsentierte wird routinemäßig „mitgesehen“ -, während Abduktionen solche eingespielten Sinnzusammenhänge eben gerade aufbrechen, um Neues zu entdecken. Die aus phänomenologischer Perspektive vorgeschlagenen Vorgehensweisen, um Abduktionen zu befördern, sind allerdings nicht auf die vorprädikative Ebene beschränkt, sondern können auch auf prädikativer Ebe- ne angewendet werden. Wie im Rahmen der Schütz'schen Ausführungen betont, sind die Grenzen zwischen den beiden „Ebenen“ sowieso fließend.

\section{$4 \quad$ Fazit}

Die Entdeckung von Neuem auf Abduktion zurückzuführen und diese als logische Operation zu konzipieren, hat etwas Faszinierendes. Noch heute evoziert man überraschte und verdutzte Gesichter, wenn man sie neben Induktion und Deduktion als das entscheidende Dritte ins Feld führt. Wie Reichertz aufzeigte, wurde indes auch Peirce immer klarer, dass Erkenntnis auf Erfahrung beruht und neben Syllogismen eine Wahrnehmungstheorie voraussetzte. Deren Konzeption ist ihm allerdings nicht sonderlich gut gelungen. Die Phänomenologie von Husserl und Schütz leisten diesbezüglich einen wesentlichen Beitrag und stellen verschiedene Prämissen des Peirce'schen Ansatzes in Frage. In kongenialer Weise liefert die Phänomenologie die epistemologischen Grundlagen für Reichertz' Vorschlag, Abduktion nicht als logische Operation zu verklären, sondern als Handlungstypus zu verstehen. Die abduktive Haltung, offen und neugierig zu sein, viel und intensiv $\mathrm{zu}$ beobachten, bereit $\mathrm{zu}$ sein sich überraschen zu lassen, prädikativ gefasste Überzeugungen einzuklammern bzw. außer Kraft zu setzen und vorprädikative Wahrnehmungen neu auszudeuten - diese abduktive Haltung lässt sich gerade auf phänomenologischer Grundlage ausgezeichnet begründen. Im Angelsächsischen würde man Reichertz wohl treffend als „,closet phenomenologist" bezeichnen.

Das Problem der Abduktion liegt darin, dass sie inhärent an eine Ontologie des Neuen gebunden bleibt. Phänomenologische Analysen der Sinnkonstitution versuchen jene formalen Invarianten zu explizieren, die immer wirksam sind, ob nun Neues konstituiert oder Altes rekonstituiert wird; ob etwas neu oder alt ist, wird einfach eingeklammert. Wie ich eingangs dargelegt habe, ist die Attribuierung von "neu“ immer relativ, einerseits aufgrund des diffusen Sprachgebrauchs, andererseits aufgrund der unterschiedlichen Bezugssysteme - was einem konkreten Subjekt neu erscheint, kann in anderen gesellschaftlichen Milieus bereits hinlänglich bekannt sein. Die phänomenologische Analyse hat gezeigt, dass sich abduktive Blitze wohl analog zu Appräsentationen ereignen, nämlich als passive Bewusstseinssynthesen. Während Appräsentationen aber auf Erfahrung beruhen, wird diese durch Abduktionen gerade durchbrochen, anders gerahmt und in neue Sinnzusammenhänge eingestellt. Allerdings wird mit der ,Entdeckung des Neuen“ ja meist stillschweigend impliziert, dass das „Neue“ ein sinnvolles Neues ist, es sich also nicht um eine „,absurde Halluzination“, eine „,ausgeflippte Spinntisiererei“ oder um ein „psychopathologisches Syndrom“" handelt. Solche Gel- 
tungssetzungen können nur innerhalb der jeweiligen gesellschaftlichen und kulturellen Relevanzsysteme vorgenommen werden, sind also relativ und indexikal.

Andererseits ist die „Entdeckung von Neuem" ein gesellschaftlich solide institutionalisiertes Relevanzsystem, zumindest seit dem Beginn der so genannten „Neuzeit“. Die Frage ist daher gesellschaftlich legitim, wie solche Prozesse gefördert werden können. Wir haben aus der Phänomenologie einige Anregungen gewonnen, wie eine abduktive Haltung praktiziert und gefördert werden kann. Dem erkenntnistheoretischen Ansatzpunkt entsprechend, beziehen sie sich alle auf das erkennende Subjekt. Dabei finden sich diverse Parallelen zu verschiedenen Kreativitätstechniken, die in der Alltagspraxis eingesetzt werden. In dieselbe Richtung zielen die zwei Großstrategien, die Peirce vorschlägt, „um das Klima für abduktive Blitze“ zu verbessern: „Verschärfen des Handlungsdrucks einerseits, vollkommene Entlastung andererseits." Beide verfolgen jedoch dasselbe Ziel: „die Ausschaltung des bewusst kontrollierenden Verstandes" (Reichertz 2003: 88). Peirce benennt damit pragmatische Bedingungen abduktiven Räsonierens. Wie eingangs erwähnt, kann diese Fragestellung auf geeignete soziale Bedingungen und organisationale Settings erweitert werden.

Reichertz $(1991,2003)$ beschäftigte sich mit Abduktion nicht nur in Bezug auf die Feldforschung, sondern auch im Rahmen der Aufklärungsarbeit von Kriminalpolizisten. Besonders verdienstvoll ist seine detaillierte und kenntnisreiche Explikation, was die drei Stufen des wissenschaftlichen Erkenntnisprozesses - Abduktion, Deduktion und Induktion - in der Forschungspraxis bedeuten. Diese Triade eignet sich, die szientifische Verkürzung der Wissenschaftstheorie auf nomologisch-deduktive Aussagensysteme zu kontern und den qualitativen Forschungsprozess in seiner ganzen Breite darzustellen. Diesem Unterfangen kommt ganz besondere Bedeutung zu in einer Zeit, in der an Universitäten wissenschaftliche Kreativität und Innovation zwar gefordert, aber nicht unbedingt gefördert werden. Wir Hochschullehrer und -lehrerinnen tun gut daran, uns immer wieder kritisch mit der Frage zu beschäftigen, unter welchen universitären Rahmenbedingungen sich eine abduktive Haltung optimal entfalten kann.

Abschließend sei angemerkt, dass die Entdeckung des Neuen per se ebenfalls ein spannendes Forschungsthema darstellt. Wie und unter welchen Bedingungen wird denn Neues entdeckt, erfunden und geschaffen? Vollziehen sich abduktive Blitze im künstlerischen Schaffen, in der Malerei oder im Komponieren von Musik auf dieselbe Weise wie in der Wissenschaft? Schütz' Analyse der mannigfaltigen Wirklichkeiten in Form unterschiedlicher Sinnbereiche legt nahe, dass dem nicht so ist. Dass neue Melodien nur auf polythetische Weise und nur auf vorprädikativer Ebene entstehen können, scheint evident - im Gegensatz etwa zu wissenschaftlichen Entdeckungen, die durchaus auch auf prädikativer Ebene stattfinden und in jedem Fall sprachlich ausformuliert werden. Der phä- nomenologische Beitrag zur Erhellung kreativer Prozesse scheint jedenfalls noch längst nicht ausgeschöpft.

\section{Literatur}

Ayim, Maryann (1974): Retroduction: The rational instinct. In: Transactions of the Charles S. Peirce Society 10: $34-43$

Duden (1989): Etymologie. Herkunftswörterbuch der deutschen Sprache. Bd. 7, 2., erw. Aufl. Mannheim/Leipzig/Wien/Zürich: Dudenverlag

Eberle, Thomas S. (1991): Rahmenanalyse und Lebensweltanalyse. In: Hettlage, Robert/Lenz, Karl (Hg.), Erving Goffman - ein soziologischer Klassiker der zweiten Generation, Bern/Stuttgart: Paul Haupt: 157-210

Eco, Umberto (1989): Introduction. In: Ogden \& Richards (1989): v-xi

Endreß, Martin/Srubar, Ilja (2003): Editorische Anmerkung E 167. In: Schütz (2003a: 175)

Génova, Gonzalo (1997): Charles S. Peirce: La lógica del descubrimiento. In: Cuadernos de Anuario Filosófico 45. Pamplona: Universidad de Navarra

Goffman, Erving (1977): Rahmenanalyse. Frankfurt am Main: Suhrkamp Verlag

Henning, Christoph (2001): Vorprädikativ. Stichwort in: Ritter et al. (2001): 1197

Husserl, Edmund (1950): Cartesianische Meditationen und Pariser Vorträge, hrsgg.v. St Strasser, Husserliana Bd.1. Den Haag: Martinus Nijhoff

Husserl, Edmund (1954): Die Krisis der Europäischen Wissenschaften und die transzendentale Philosophie, hrsg. v. W. Biemel, Husserliana Bd. 6. Den Haag: Martinus Nijhoff

Husserl, Edmund (1971, 1976, 1991): Ideen zu einer reinen Phänomenologie und phänomenologischen Philosophie. Erstes Buch, hrsg. v. K. Schuhmann, Husserliana Bd. 3 (1976). Zweites Buch, hrsg. v. M. Biemel, Husserliana Bd. 4 (1991). Drittes Buch, hrsg.v. W. Biemel, Husserliana Bd. 6 (1971). Den Haag: Martinus Nijhoff

Husserl, Edmund (1985): Erfahrung und Urteil. Untersuchungen zur Genealogie der Moral, hrsg.v. L. Landgrebe. Hamburg: Meiner

Knoblauch, Hubert/Kurt, Ronald/Soeffner (2003), Hans-Georg; Einleitung der Herausgeber: Zur kommunikativen Ordnung der Lebenswelt. Alfred Schütz' Theorie der Zeichen, Sprache und Kommunikation. In: Schütz (2003b): 7-33

Kreativitätstechniken. Gefunden am 1.1.11 unter: http://www.creajour.de/methodisches/technikglossar/index.html

Mullin, Albert A. (1966): C.S.S. Peirce and E.G.A. Husserl on the Nature of Logic. In: Notre Dame Journal of Formal Logic VII. 4: 301-304

Nubiola, Jaime (2003). The Abduction of God. To appear in C. Pearson (ed.): Progress in Peirce Studies: Religious Writings - Gefunden am 1.1.11 unter: http://www. unav.es/users/AbductionOfGod.html

Nubiola, Jaime (2005): Abduction or the Logic of Surprise. In: Semiotica 153. 117-130

Ogden, Charles K./Richards, Ivor A. (1989): The Meaning of Meaning. San Diego: Harcourt 
Peirce, Charles Sanders (1908): The Collected Papers of Charles S. Peirce. Vol. 6 C.Hartshorne, P. Weiss and A.W. Burks (eds.). Cambridge University Press.

Peirce, Charles Sanders (1976 (1967/1970)): Schriften zum Pragmatismus und Pragmatizismus, hrsg.v. K.-O. Apel und übers. v. G. Wartenberg

Petrilli, Susan (2010): Image and Primary Iconism: Perice and Husserl. In: Semiotica 181 263-274

Reichertz, Jo (1991): Aufklärungsarbeit. Kriminalpolizisten und Feldforscher bei der Arbeit. Stuttgart: Enke

Reichertz, Jo (2003): Die Abduktion in der qualitativen Sozialforschung. Opladen: Leske + Budrich

Renn, Joachim/Sebald, Gerd/Weyand, Jan (im Druck): Lebenswelt und Lebensform. Weilerswist: Velbrück Wissenschaft

Ritter, Joachim/Gründer, Karlfried/Gabriel, Gottfried (2001): Historisches Wörterbuch der Philosophie. Bd. 11. Basel: Schwabe \& Co

Russell, Bertrand (1959): Wisdom of the West. Garden City, N.Y.: Doubleday

Scheler, Max (1926): Die Wissensformen und die Gesellschaft. Leipzig: Der Neue Geist

Schütz, Alfred (2003a): Theorie der Lebenswelt 1. Die pragmatische Schichtung der Lebenswelt. ASW V.1. Konstanz: UVK

Schütz, Alfred (2003b): Theorie der Lebenswelt 2. Die Kommunikative Ordnung der Lebenswelt. ASW V.2. Konstanz: UVK

Schütz, Alfred (2004a): Der sinnhafte Aufbau der sozialen Welt. Eine Einleitung in die verstehende Soziologie. ASW II. Konstanz: UVK

Schütz, Alfred (2004b): Relevanz und Handeln 1. Zur Phänomenologie des Alltagswissens. ASW VI.1. Konstanz: UVK

Spiegelberg, Herbert (1956): Husserl's and Peirce's Phenomenologies: Coincidence or Interaction. In: Philosophy and Phenomenological Research 17. 2: 164-185

Srubar, Ilja (1988): Kosmion. Die Genese der pragmatischen Lebenswelttheorie von Alfred Schütz und ihr anthropologischer Hintergrund. Frankfurt am Main: Suhrkamp Verlag

Srubar, Ilja (2007): Phänomenologische und soziologische Theorie. Aufsätze zur pragmatischen Lebenswelttheorie. Wiesbaden: VS Verlag für Sozialwissenschaften

Srubar, Ilja (im Druck): Formen asemiotischer Kommunikation, in: Joachim Renn/Gerd Sebald/Jan Weyand (im Druck)

Stjernfeldt, Frederik (2007): Diagrammatology: An Investigation on the Borderlines of Phenomenology, Ontology and Semiotics. Wien/New York: Springer

Watzlawick, Paul (Hg.) (1981): Die erfundene Wirklichkeit. Wie wissen wir, was wir zu wissen glauben? München: Piper 